TRANSACTIONS OF THE

AMERICAN MATHEMATICAL SOCIETY

Volume 358, Number 2, Pages 687-702

S 0002-9947(05)03634-2

Article electronically published on March 10, 2005

\title{
EQUIVARIANT GYSIN MAPS AND PULLING BACK FIXED POINTS
}

\author{
BERNHARD HANKE AND VOLKER PUPPE
}

Dedicated to William Browder on the occasion of his 70th birthday

\begin{abstract}
We develop a new approach to the pulling back fixed points theorem of $\mathrm{W}$. Browder and use it in order to prove various generalizations of this result.
\end{abstract}

\section{Introduction}

In [3, W. Browder proves

Theorem 1. Let $G$ be a finite abelian p-group, let $M$ be an oriented smooth $G$ manifold and let $N$ be an oriented PL G-manifold. Assume that $M$ and $N$ are without boundary and have the same dimension. Let

$$
f: M \rightarrow N
$$

be a continuous proper $G$-equivariant map. Additionally, assume that if $p=2$ and $H<G$, then the normal bundle of $M^{H}$ in $M$ has the structure of a complex linear $G$-bundle. Then, if the degree of $f$ is not divisible by $p$, the induced map of fixed point sets

$$
f^{G}: M^{G} \rightarrow N^{G}
$$

is surjective (put differently, any point in $N^{G}$ can be pulled back under $f$ to a point in $M^{G}$ ).

Actually, the main result, Theorem (1.1) in [3], says that under the above conditions, the induced map in $\mathbb{F}_{p^{\text {-cohomology }}}$

$$
\left(f^{G}\right)^{*}: H^{*}\left(N^{G} ; \mathbb{F}_{p}\right) \rightarrow H^{*}\left(M^{G} ; \mathbb{F}_{p}\right)
$$

is injective. The conclusion stated above follows from this fact.

As a corollary of Theorem 1 Browder shows that a finite abelian $p$-group cannot act smoothly on an oriented closed manifold with exactly one fixed point (with the extra assumption, if $p=2$ ). This was proved independently by Ewing and Stong in [7] who also characterize those finite groups that can act smoothly on a closed manifold with exactly one fixed point. In particular, they show that for any finite group which is not abelian or contains elements of coprime order, such actions exist

Received by the editors October 24, 2003 and, in revised form, February 19, 2004.

2000 Mathematics Subject Classification. Primary 55N20, 55N91, 57S17; Secondary 57N65.

Key words and phrases. Group action, generalized homology, topological manifold.

The first author is a member of the European Differential Geometry Endeavour (EDGE), Research Training Network HPRN-CT-2000-00101, supported by The European Human Potential Programme. 
(even if one requires the underlying manifold to be oriented and the action to be orientation preserving). A concrete example of such with a group of order $p^{3}$ acting on a complex projective space is also described in Browder's paper. In particular, Theorem 1 cannot be extended to a larger class of finite groups.

Theorem 1 was shown by an involved argument relying heavily on the differentiability of the action on $M$. On the other hand, if $G$ is an elementary abelian $p$-group, it is well known that this theorem holds more generally for actions on $\mathbb{F}_{p}$-homology manifolds. The proof of this last result uses equivariant (ordinary) cohomology and the localization theorem [1, 2], standard techniques in Smith theory.

As Browder points out at the end of his paper, it would be desirable to prove versions of his theorem in more general contexts, above all to weaken the differentiability assumption on $M$.

In this paper, we will provide such generalizations and in particular, if $G$ is a finite cyclic $p$-group, we remove the assumption of differentiability of the $G$-action on $M$. Our approach is different from Browder's. It is based on a combination of the Atiyah-Segal-tom Dieck localization theorem with equivariant Gysin maps for generalized equivariant cohomology theories. In this respect, our discussion provides a link of Browder's result to methods building on classical Smith theory.

The authors are grateful to $\mathrm{W}$. Browder for useful discussions about the subject treated in this paper and to S. Mardešić and Y. Rudyak for helpful comments concerning Čech-extensions of and Poincaré duality with respect to generalized (co-)homology theories.

\section{MAIN RESUltS}

Let $G$ be a finite group and let $E$ be a pointed complex oriented ring spectrum. Then $E$ induces homology and cohomology theories defined on $C W$-pairs as usual. The cohomology theory $E^{*}$ has a Čech extension (denoted by $E^{*}$ again) defined on pairs of arbitrary topological spaces (cf. 14]) such that the following tautness assumption is satisfied: If $X$ is a paracompact space and $A \subset X$ is a closed subspace, we have

$$
E^{*}(X, A)=\lim _{A \subset U} E^{*}(X, U)
$$

where $U$ runs over all open neighbourhoods of $A$ in $X$.

Let $E G \rightarrow B G$ be the universal $G$-fibration, where $E G$ is considered as a right $G$-space. The Borel construction

$$
X \mapsto X_{G}=E G \times_{G} X,(f: X \rightarrow Y) \mapsto f_{G}=\mathrm{id} \times_{G} f: X_{G} \rightarrow Y_{G},
$$

defines a functor from the category of $G$-spaces and $G$-maps to the category of spaces and maps, and so we get an associated stable equivariant cohomology theory $E_{G}^{*}$ (graded over the integers) in the sense of [4, Def. (II.6.8), by setting

$$
E_{G}^{*}(X)=E^{*}\left(X_{G}\right) \text {, etc. }
$$

Using the complex orientation of $E$, we consider the multiplicative subset

$$
S \subset E^{*}(B G)
$$

consisting of Euler classes of complex $G$-representations without trivial direct summand (each complex $G$-representation $V$ gives rise to a complex vector bundle $\left.E G \times_{G} V \rightarrow B G\right)$.

The following version of the localization theorem (which might be of independent interest) will be shown by a slight modification of the discussion in [4, (III.3). We 
remark that our proof is independent of the equivariant tautness assumption stated in [4], Def. (III.3.5), which is not clear for our theory $E_{G}^{*}$.

Theorem 2. Let $X$ be a paracompact $G$-space of finite covering dimension. Then the inclusion $X^{G} \hookrightarrow X$ induces an isomorphism

$$
S^{-1} E_{G}^{*}(X) \cong S^{-1} E_{G}^{*}\left(X^{G}\right) .
$$

Throughout the paper (and if not indicated otherwise), the term $G$-manifold stands for a closed topological manifold $X$ equipped with a topological $G$-action such that $X$ is a $G$-ANR. By the well-known results from [9], this additional requirement is certainly fulfilled, if either the action on $X$ is locally linear or $X$ is triangulable and $G$ acts by simplicial maps. We remind the reader of the fact that the fixed point set of a simplicial group action on a topological manifold need not be a topological manifold.

Let $M$ be a $G$-manifold. By applying the Borel construction to the total space $T M=M \times M$ (equipped with the diagonal $G$-action) and the base space $M$ of the tangent microbundle of $M$, we get an induced microbundle $(T M)_{G}$ over $M_{G}$ (note that the induced diagonal $G$-actions on $E G \times(M \times M)$, resp. on $E G \times M$ are free).

Definition 1. By an $E_{G}$-orientation of $M$, we mean an $E$-orientation, i.e. a (generalized) Thom class, of the microbundle $(T M)_{G}$ over $M_{G}$ in the nonequivariant sense (cf. [17, Def. 14.5).

By restriction to the fibre in the Borel construction, it is clear that any $E_{G^{-}}$ orientation of $M$ induces an $E$-orientation of $M$.

Let $M$ and $N$ be $E_{G}$-oriented $G$-manifolds of the same dimension. Let $f: M \rightarrow N$ be a continuous $G$-map. Then $f$ induces a (degree preserving) Gysin map $f_{!}$: $E^{*}(M) \rightarrow E^{*}(N)$ (cf. Section 4). The element $f_{!}(1) \in E^{0}(N)$ (where $1 \in E^{0}(M)$ is the canonical unit) is called the $E$-degree of $f$. Using Theorem 2 and the equivariant Gysin map constructed in Section 4 , we get the following analogue of Browder's injectivity result stated at the beginning of this paper.

Theorem 3. If the E-degree $f_{!}(1)$ is a unit in $E^{0}(N)$, then $f$ induces a split injection of $S^{-1} E^{*}(B G)$-modules

$$
S^{-1} E^{*}\left(B G \times N^{G}\right) \rightarrow S^{-1} E^{*}\left(B G \times M^{G}\right) .
$$

This theorem contains useful information, only if the left-hand side is different from zero. This can only happen if $E$ has the property described in the next definition.

Definition 2. The theory $E$ is called $G$-sensitive if

$$
S^{-1} E^{*}(B G) \neq 0 .
$$

Typical examples of $G$-sensitive theories are Čech cohomology with $\mathbb{F}_{p^{-}}$ coefficients for elementary abelian $p$-groups [1], complex $K$-theory for cyclic $p$ groups (Lemma 4) and unitary bordism for finite abelian $p$-groups [5]. On the other hand Čech cohomology with integer or $\mathbb{F}_{p}$-coefficients is not $\mathbb{Z} / p^{2}$-sensitive and complex $K$-theory is not $G$-sensitive, if $G=\mathbb{Z} / p \times \mathbb{Z} / p$ or $G=\mathbb{Z} / n$, if $n>1$ is not a prime power. For nonabelian $G$, none of these theories is $G$-sensitive (cf. [5], proof of Satz 3). 
Theorem 4. Let $M$ and $N$ be $E_{G}$-orientable $G$-manifolds of the same dimension, where $E$ is $G$-sensitive. Furthermore, let $M$ and $N$ be oriented in the usual sense and let $f: M \rightarrow N$ be a continuous $G$-map of ordinary degree $d \in \mathbb{Z}$. If $d \cdot 1 \in E^{0}$ is a unit, then the induced map of fixed points

$$
f^{G}: M^{G} \rightarrow N^{G}
$$

is surjective.

Theorem 4 gains its power by judicuous choices of the theory $E$. Using unitary bordism, we essentially reprove Browder's result. On the other hand, with the use of complex $K$-theory, we obtain the following generalization of Browder's theorem in the case of actions of cyclic $p$-groups.

Theorem 5. Let $G$ be a finite cyclic p-group, where $p$ is odd. Let $M$ and $N$ be oriented (in the usual sense) $G$-manifolds of the same dimension. If $f: M \rightarrow N$ is a $G$-map whose (nonequivariant) degree is not a multiple of $p$, then the induced map of fixed point sets $f^{G}: M^{G} \rightarrow N^{G}$ is surjective.

We remark that this theorem would hold for any finite abelian $p$-group ( $p$ odd), if the following conjecture is true.

Conjecture. There exists a complex oriented cohomology theory $E^{*}$ with the following properties: Every closed oriented manifold is orientable with respect to $E^{*}$ and $E^{*}$ is $G$-sensitive for every finite abelian $p$-group $G$, where $p$ is an odd prime.

If $E$ were a cohomology theory with these properties, it would follow from the proof of Lemma 5 that if $X$ were a $G$-manifold which was orientable in the usual

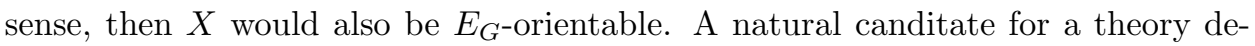
scribed in this conjecture is topological bordism, for which the first property is immediate. Unfortunately, it seems to be beyond present knowledge, if this theory is also $G$-sensitive for every finite abelian $p$-group $G$ (where $p$ is odd). Nevertheless, it is obvious that topological bordism is $G$-sensitive if $G$ is a cyclic $p$-group or if $G$ is an elementary abelian $p$-group: We have both Sullivan's $K$-theory orientation and the ordinary homology orientation of the oriented bordism spectrum localized at an odd prime. This gives some indication that the above conjecture might indeed be true.

The difficulty that a theory is not $G$-sensitive can sometimes be circumvented by an inductive procedure based on the ideas of the proof of Theorem 3 , This will be illustrated in the last part of this paper, where we sketch a third approach to Browder's result using classical Smith theory. Moreover, assuming some strong cohomological restrictions on the spaces involved, we even can remove the assumption that $G$ is abelian:

Theorem 6. Let $M$ and $N$ be compact oriented $\mathbb{F}_{p}$-cohomology manifolds of the same dimension (we use Čech-cohomology), let $G$ be a finite p-group and assume the following: The $\mathbb{F}_{p}$-cohomology of $M$ and $N$ is concentrated in even degrees and

$$
p>\max \left\{\operatorname{dim}_{\mathbb{F}_{p}} H^{*}\left(M ; \mathbb{F}_{p}\right), \operatorname{dim}_{\mathbb{F}_{p}} H^{*}\left(N ; \mathbb{F}_{p}\right)\right\} .
$$

Let $f: M \rightarrow N$ be $G$-equivariant. If the degree of $f$ is a nonzero number in $\mathbb{F}_{p}$, then the induced map $M^{G} \rightarrow N^{G}$ is surjective. 


\section{LOCALIZATION THEOREM}

In this short section, we will prove Theorem 2 above. Our proof will be modelled on the proof of Theorem (III.3.6) in [4]. We set $A=X^{G}$. At first, notice that by our assumptions and because $G$ is finite, $X-A$ is of finite $S$-type in the sense of 4, Def. (III.3.2). We now have to show that

$$
S^{-1} E_{G}^{*}(X, A)=0 .
$$

Using the (nonequivariant) tautness of $E^{*}$, it is enough to show that

$$
S^{-1} E^{*}\left(X_{G}, U\right)=0,
$$

for all open $A_{G} \subset U \subset X_{G}$. At this point, we remark that the projection $X_{G} \rightarrow B G$ restricts to a map $U \rightarrow B G$ so that $E^{*}(U)$ has indeed the structure of an $E^{*}(B G)$ module. By excision

$$
S^{-1} E^{*}\left(X_{G}, U\right) \cong S^{-1} E^{*}\left(X_{G}-A_{G}, U-A_{G}\right) .
$$

Because $X-A$ is of finite $S$-type, we have

$$
S^{-1} E^{*}\left((X-A)_{G}\right)=0
$$

by [4, Theorem (III.3.3), and because the inclusion $U-A_{G} \hookrightarrow(X-A)_{G}$ induces a map of $E^{*}(B G)$-algebras $E^{*}\left((X-A)_{G}\right) \rightarrow E^{*}\left(U-A_{G}\right)$, this implies that also

$$
S^{-1} E^{*}\left(U-A_{G}\right)=0 .
$$

The long exact cohomology sequence gives

$$
S^{-1} E^{*}\left(X_{G}-A_{G}, U-A_{G}\right)=0,
$$

and this completes the proof of Theorem 2 .

A relative version of Theorem 2 follows easily by use of the long exact cohomology sequence. It is also obvious that we could have proven a version of Theorem 2 for infinite groups $G$, but we restricted ourselves to the case treated here in view of our later applications.

\section{EQUivariant GySin MAPS}

Let $G$ be a finite group, let $M$ and $N$ be $E_{G}$-oriented $G$-manifolds of dimension $m$ and $n$, respectively. Let $f: M \rightarrow N$ be a continuous $G$-equivariant map. We will construct an equivariant Gysin map

$$
\left(f_{G}\right)_{!}: E_{G}^{k}(M) \rightarrow E_{G}^{k+n-m}(N), k \in \mathbb{Z},
$$

along the lines of [10]. By [15], there is a $G$-embedding $M \rightarrow V$ into a complex $G$-representation. Let $c$ be its complex dimension. Using the map $f$ and this embedding, we consider $M$ as a $G$-submanifold of $N \times V$ (equipped with the diagonal $G$-action). In a first step, we need to construct a Thom-like class

$$
\tau \in E^{n+2 c-m}\left((N \times V)_{G},(N \times V)_{G}-M_{G}\right)
$$

which is straightforward if $M$ has a (stable) normal $G$-microbundle in $N \times V$. Because the literature (cf. [8]) provides such a bundle, only if the $G$-spaces under consideration are locally linear, we proceed as follows.

As $M$ is a $G$-ANR (by our definition of $G$-manifold), there is a $G$-invariant neighbourhood $U \subset N \times V$ of $M$ and a $G$-equivariant retraction

$$
r: U \rightarrow M \text {. }
$$


We choose a $G$-CW model of $E G$ and obtain a skeletal filtration

$$
E G^{0} \subset E G^{1} \subset E G^{2} \subset \ldots
$$

of $E G$. Write $M_{G}^{i}$ for $E G^{i} \times_{G} M$ and so forth. At first, we construct a compatible sequence of Thom classes

$$
\tau^{i} \in E^{n+2 c-m}\left((N \times V)_{G}^{i},(N \times V)_{G}^{i}-M_{G}^{i}\right) .
$$

Using the $E_{G}$-orientation of $N$ and the canonical $E_{G}$-orientation of $V$, we get an $E_{G}$-orientation of the $G$-manifold $N \times V$. By restriction, we then get $E$-orientations of the microbundles $(T M)_{G}^{i}$ over $M_{G}^{i}$ and of $(T(N \times V))_{G}^{i}$ over $(N \times V)_{G}^{i}$.

We will construct a normal microbundle $\nu$ of $M_{G}^{i}$ in $(N \times V)_{G}^{i} \times \mathbb{R}^{q}$ for some $q$ along the lines of 12. By this, we mean that (the total space of) $\nu$ is homeomorphic to a neighbourhood of $M_{G}^{i}$ in $(N \times V)_{G}^{i} \times \mathbb{R}^{q}$ by a homeomorphism mapping the zero section of $\nu$ to $M_{G}^{i}$.

The retraction $r$ induces a retraction

$$
r_{G}^{i}: U_{G}^{i} \rightarrow M_{G}^{i} .
$$

Denoting a microbundle and its total space with the same letter, the proof of [12], Lemma (5.3), can be carried out fibrewise in the Borel fibration and shows that we have a homeomorphism

$$
\left.(T U)_{G}^{i}\right|_{M_{G}^{i}} \approx\left(r_{G}^{i}\right)^{*}(T M)_{G}^{i} .
$$

In particular, the total space of $\left.(T U)_{G}^{i}\right|_{M_{G}^{i}}$ constitutes a microbundle neighbourhood (defined in the obvious sense) of $M_{G}^{i}$ in $\left(r_{G}^{i}\right)^{*}(T M)_{G}^{i}$ (cf. [12, Lemma (5.4)). The space $M_{G}^{i}$ being compact, there is a stably unique microbundle $\eta$ over $M_{G}^{i}$ complementary to $(T M)_{G}^{i}$, i.e. its Whitney sum with $(T M)_{G}^{i}$ is a trivial bundle $\underline{\mathbb{R}}^{q}$. As in 12 , Theorem (5.8), the bundle $\eta$ can be used in order to construct a normal microbundle $\nu$ of the required sort.

Theorem (5.9) in 12 can also be adapted to our situation and shows that we get a microbundle isomorphism

$$
\left.(T M)_{G}^{i} \oplus \nu \cong(T(N \times V))_{G}^{i}\right|_{M_{G}^{i}} \oplus \underline{\mathbb{R}^{q}} .
$$

For a translation of the last part of the proof of Theorem (5.9) in [12, choose a $G$-invariant neighbourhood $D$ of the diagonal in $M \times M$ such that $\left.p_{1}\right|_{D}$ and $\left.p_{2}\right|_{D}$ (where $p_{1}$ and $p_{2}$ are projections) are equivariantly homotopic (this can be done because $M$ is a $G$-ANR) and apply the Borel construction to $D$.

By [6], the microbundle $\nu$ has a canonical $E$-orientation. The class $\tau^{i}$ is the $q$-fold desuspension of the Thom class of this bundle.

We want to show that under the inclusion $M_{G}^{i} \subset M_{G}^{i+1}$, the Thom class $\tau^{i+1}$ is mapped to $\tau^{i}$. But this is obvious, because a complementary bundle to $(T M)_{G}^{i+1}$ restricted to $M_{G}^{i}$ is complementary to $(T M)_{G}^{i}$, and the rest of the construction of the normal bundle $\nu$ above was based on data which were independent of $i$.

Because the canonical map

$$
\begin{aligned}
\rho: E^{n+2 c-m}\left((N \times V)_{G},(N \times V)_{G}-M_{G}\right) & \\
& \rightarrow \lim _{i} E^{n+2 c-m}\left((N \times V)_{G}^{i},(N \times V)_{G}^{i}-M_{G}^{i}\right)
\end{aligned}
$$

in the Milnor exact sequence is surjective, we can choose a class

$$
\tau \in E^{n+2 c-m}\left((N \times V)_{G},(N \times V)_{G}-M_{G}\right)
$$


restricting to the inverse system $\left(\tau^{i}\right)$. But notice that due to the possible nonvanishing of $\lim ^{1}$, this class need not be uniquely determined by $\left(\tau^{i}\right)$.

Multiplication with $\tau$ from the right induces a map

$$
\begin{aligned}
\phi: E^{k}\left(M_{G}\right) \stackrel{r_{G}^{*}}{\rightarrow} E^{k}\left(U_{G}\right) \stackrel{\cdot \tau}{\rightarrow} E^{k+n+2 c-m}\left(U_{G}, U_{G}-M_{G}\right) \\
\quad \cong E^{k+n+2 c-m}\left((N \times V)_{G},(N \times V)_{G}-M_{G}\right) .
\end{aligned}
$$

This is an isomorphism, because it induces isomorphisms

$$
E^{k}\left(M_{G}^{i}\right) \cong E^{k+n+2 c-m}\left((N \times V)_{G}^{i},(N \times V)_{G}^{i}-M_{G}^{i}\right)
$$

for all $i$ (and hence induces isomorphisms of the $\lim ^{0}$ and $\lim ^{1}$ terms in the respective Milnor exact sequences). In this respect, we might consider $\tau$ as a Thom class.

Now choose a $G$-invariant disc $D(V) \subset V$ such that $M \subset N \times D(V)$ and denote its boundary by $S(V)$. The equivariant Gysin map $\left(f_{G}\right)$ ! is defined as the composition

$$
\begin{aligned}
E^{k}\left(M_{G}\right) & \stackrel{\phi}{\rightarrow} E^{k+n+2 c-m}\left((N \times V)_{G},(N \times V)_{G}-M_{G}\right) \\
& \rightarrow E^{k+n+2 c-m}\left((N \times D(V))_{G},(N \times S(V))_{G}\right) \\
& \rightarrow E^{k+n-m}\left(N_{G}\right),
\end{aligned}
$$

where the first map is the Thom isomorphism constructed before, the second map is induced by an inclusion of spaces and the last map is the inverse of the Thom isomorphism of the complex vector bundle

$$
V \hookrightarrow(N \times V)_{G} \rightarrow N_{G} .
$$

The following properties of $\left(f_{G}\right)$ ! are proven similarly as in [10]:

- Under restriction to the fibres in the Borel constructions, $\left(f_{G}\right)$ ! restricts to the usual Gysin map

$$
f_{!}: E^{k}(M) \rightarrow E^{k+n-m}(N),
$$

where we use the induced $E$-orientations of $M$ and $N$. Recall that $f_{!}$is induced by the map in homology $f_{*}: E_{*}(M) \rightarrow E_{*}(N)$ and the Poincaré duality isomorphisms for $M$ and $N$.

- $\left(f_{G}\right)$ ! is an $E^{*}(B G)$-module map.

- For $x \in E_{G}^{*}(N)$, we have

$$
\left(f_{G}\right) !\left(f_{G}^{*}(x)\right)=x \cup\left(f_{G}\right)_{!}(1) .
$$

From now on, we assume that $m=n$.

Lemma 1. If the $E$-degree $f_{!}(1) \in E^{0}(N)$ is a unit, then the composition

$$
E_{G}^{*}(N) \stackrel{f_{G}^{*}}{\rightarrow} E_{G}^{*}(M) \stackrel{\left(f_{G}\right) !}{\rightarrow} E_{G}^{*}(N)
$$

is an isomorphism.

Proof. We use a spectral sequence argument. The Borel fibrations for $M$ and $N$ lead to converging Atiyah-Hirzebruch spectral sequences ([17], Theorem 15.7)

$$
E_{2}^{p, q}(M)=H^{p}\left(B G ; E^{q}(M)\right) \Longrightarrow E_{G}^{p+q}(M)
$$

and similarly for $N$. Clearly, the map $\left(f_{G}\right)^{*}$ induces a map of $E_{2}$-terms. But also $\left(f_{G}\right)$ ! induces a map of $E_{2}$-terms

$$
H^{p}\left(B G ; E^{q}(M)\right) \rightarrow H^{p}\left(B G ; E^{q}(N)\right)
$$


which is induced by the nonequivariant Gysin map $f !: E^{q}(M) \rightarrow E^{q}(N)$. Hence, on the $E_{2}$-level, the composition $\left(f_{G}\right) ! \circ\left(f_{G}\right)^{*}$ is induced by the map of coefficients

$$
E^{q}(N) \stackrel{f^{*}}{\rightarrow} E^{q}(M) \stackrel{f_{1}}{\rightarrow} E^{q}(N) .
$$

This map is given by $x \mapsto x \cup f_{!}(1)$ and hence is an isomorphism by our assumption. Consequently, the induced map of $E_{\infty}$-terms is an isomorphism, too. This implies our assertion.

In order to start with the proof of Theorem 3, we consider the multiplicative subset $S \subset E_{G}^{*}=E^{*}(B G)$ generated by Euler classes of nontrivial irreducible complex $G$-representations. Theorem 2 yields a commutative localization diagram

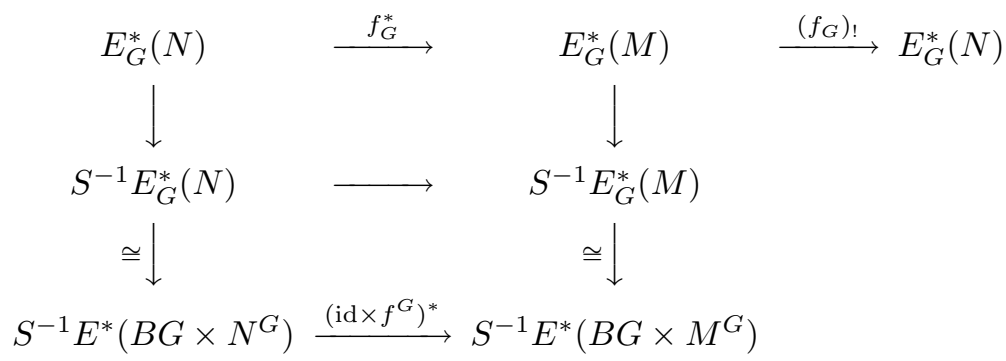

The first line is an isomorphism by Lemma 1 and in particular, the map $f_{G}^{*}$ is a split injection of $E^{*}(B G)$-modules. This is therefore also true for the map (id $\left.\times f^{G}\right)^{*}$ in the third line. Hence, the proof of Theorem 3 is complete.

Now additionally assume that $M$ and $N$ are oriented in the usual sense.

Lemma 2. Let $d \in \mathbb{Z}$ be the usual degree of $f$. If $d \cdot 1 \in E^{0}$ is a unit, then $f_{!}(1) \in E^{0}(N)$ is also a unit.

Proof. If $X$ is a closed oriented topological manifold of dimension $n$, obstruction theory yields a canonical bijection between the set $\left[X, S^{n}\right]$ of homotopy classes of maps $X \rightarrow S^{n}$ and elements of $H^{n}(X ; \mathbb{Z}$ ) (recall that $X$ is homotopy equivalent to a $C W$-complex). Let $\pi: M \rightarrow S^{n}$ and $\psi: N \rightarrow S^{n}$ be maps of degree 1 (given by collapsing the exterior of a small ball to a point). If we take a map $S^{n} \rightarrow S^{n}$ of degree $d$, we hence get a diagram

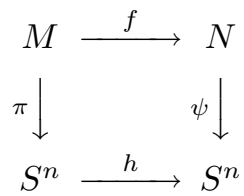

commuting up to homotopy. We can choose orientation classes $[M] \in \tilde{E}_{n}(M)$ and $[N] \in \tilde{E}_{n}(N)$ such that $\pi_{*}([M])=\left[S^{n}\right]=\psi_{*}([N])$, where $\left[S^{n}\right] \in \tilde{E}_{n}\left(S^{n}\right)$ denotes the canonical orientation class (i.e. the $n$-fold suspension of $1 \in \tilde{E}_{0}\left(S^{0}\right)$ ). In particular, we have $(h \circ \pi)_{*}[M]=d \cdot\left[S^{n}\right]$. Because $(h \circ \pi)_{!}(1)$ is the Poincaré dual of $(h \circ \pi)_{*}([M])$, we get the equation

$$
\psi_{!} \circ f_{!}(1)=d \cdot 1 \in E^{0} \subset E^{0}\left(S^{n}\right) .
$$

We have canonical splittings

$$
E^{0}(N)=\tilde{E}^{0}(N) \oplus E^{0}, E^{0}\left(S^{n}\right)=\tilde{E}^{0}\left(S^{n}\right) \oplus E^{0}
$$


and by Alexander duality ([17, Theorem 14.11) we see that $\psi_{\text {! }}$ induces a map of reduced groups $\tilde{E}^{0}(N) \rightarrow \tilde{E}^{0}\left(S^{n}\right)$. Altogether, this implies that the component of $f_{!}(1)$ in $E^{0}$ must be equal to $d \cdot 1$. Because $N$ is finite dimensional, $\tilde{E}^{0}(N)$ is nilpotent (apply the Atiyah-Hirzebruch spectral sequence) and the element $f_{!}(1) \in E^{0}(N)$ is indeed a unit.

For the proof of Theorem 4 , choose a point $y \in N^{G}$ and assume that $f^{-1}(y) \cap$ $M^{G}=\emptyset$. This implies that the composition

$$
\chi: S^{-1} E^{*}\left(B G \times\left(N^{G}, N^{G}-\{y\}\right)\right) \rightarrow S^{-1} E^{*}\left(B G \times N^{G}\right) \rightarrow S^{-1} E^{*}\left(B G \times M^{G}\right)
$$

is zero.

Let $\pi: N \rightarrow\{y\}$ be the unique $G$-map and consider the composition of $E^{*}(B G)$ linear maps

$$
E^{*}\left(N_{G},(N-\{y\})_{G}\right)=E_{G}^{*}(N, N-\{y\}) \rightarrow E_{G}^{*}(N) \stackrel{\left(\pi_{G}\right) !}{\rightarrow} E_{G}^{*}(\{y\})=E^{*}(B G),
$$

where $\left(\pi_{G}\right)$ ! is the equivariant Gysin map considered before. This composition decreases the degree by $n$. The pair $\left(N_{G},(N-\{y\})_{G}\right)$ can be considered as the Thom space of the restricted bundle $\left(\left.T N\right|_{\{y\}}\right)_{G}$. Because $N$ is $E_{G^{-}}$orientable, this restricted bundle is $E$-orientable and the composition under consideration is an isomorphism: It follows directly from the construction of Gysin maps at the beginning of Section 4 that for each $i$, the corresponding map

$$
E^{*}\left(N_{G}^{i},(N-\{y\})_{G}^{i}\right) \rightarrow E^{*}\left(B G^{i}\right)
$$

is exactly the inverse of the Thom isomorphism of the $E$-oriented bundle $\left(\left.T N\right|_{\{y\}}\right)_{G}^{i}$ and therefore is an isomorphism. An application of the Milnor exact seqence to the induced isomorphism of inverse systems shows that the composition

$$
E^{*}\left(N_{G},(N-\{y\})_{G}\right) \rightarrow E^{*}(B G)
$$

is an isomorphism, as well. In particular, the induced localized map

$$
S^{-1} E^{*}\left(N_{G},(N-\{y\})_{G}\right) \rightarrow S^{-1} E^{*}(B G)
$$

is surjective. Its image is different from zero, because $E$ is $G$-sensitive. Application of the localization theorem finally shows that the image of the first map in the composition $\chi$ is not zero.

By Lemma 2 and Theorem 3, the second map in this composition is injective.

Hence, we get a contradiction to the fact that the composition $\chi$ is zero. The assumption $f^{-1}(y) \cap M^{G}=\emptyset$ must therefore be false and the proof of Theorem 4 is complete.

\section{Applications and examples}

The first choice for $E$ that comes to mind is Cech cohomology with $\mathbb{F}_{p}$-coefficients. If $G$ is an elementary abelian $p$-group, $M$ and $N$ are closed $G$-manifolds which are oriented, if $p$ is odd, and if $f: M \rightarrow N$ is a $G$-equivariant map whose degree (as an element in $\mathbb{F}_{p}$ ) is different from 0 , then all the assumptions stated in Theorem 4 are fulfilled and the induced map of fixed point sets is surjective. It is well known that this conclusion holds as well if $M$ and $N$ are just assumed to be compact oriented $\mathbb{F}_{p}$-homology manifolds. Also, note that the conclusion of Theorem 3 holds, if we just assume that $M$ and $N$ are compact $\mathbb{F}_{p}$-Poincaré duality spaces. In particular, the induced map $H^{*}\left(N^{G} ; \mathbb{F}_{p}\right) \rightarrow H^{*}\left(M^{G} ; \mathbb{F}_{p}\right)$ is injective in this case. 
Now let $G$ be a finite abelian $p$-group. For $E$, we take the unitary bordism spectrum localized at $p$ (and denoted by $M U_{(p)}$ ). It follows from results of tom Dieck [5] that $M U_{(p)}$ is $G$-sensitive.

Lemma 3. Let $p$ be odd and let $M$ be an oriented closed smooth $G$-manifold. Then

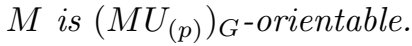

Proof. For odd $p$, we have an orientation (i.e. a map of ring spectra)

$$
\mathrm{MSO}_{(p)} \rightarrow M U_{(p)},
$$

cf. [13] or [16. In particular, any oriented vector bundle is $M U_{(p)}$-orientable. If $M$ is an oriented smooth $G$-manifold, the induced bundle $(T M)_{G} \rightarrow M_{G}$ is orientable and therefore $M U_{(p)}$-orientable.

If $p=2$, one shows similarly that $M$ is $\left(M U_{(p)}\right)_{G}$-orientable, if $M$ is a stably almost complex manifold and the group action preserves the stable almost complex structure of $M$. Using Lemma 3 and Theorem 4 applied to $E=M U_{(p)}$, we get the following version of Browder's original theorem.

Theorem 7. Let $G$ be a finite abelian p-group. Let $M$ and $N$ be closed oriented smooth $G$-manifolds if $p$ is odd, or closed stably complex $G$-manifolds if $p=2$. Assume that $M$ and $N$ have the same dimension. If $f: M \rightarrow N$ is an equivariant map whose degree is not divisible by $p$, then the induced map $M^{G} \rightarrow N^{G}$ is surjective.

Note that for reproving Browder's theorem (in the differentiable case), we need not refer to the construction of equivariant Gysin maps in Section 4, but can use directly the corresponding results from [10].

The following well-known example (cf. [7) shows that the case $p=2$ really requires special attention: The action of $\mathbb{Z} / 4$ on $\mathbb{C} P^{2}$ induced by

$$
\left[x_{0}: x_{1}: x_{2}\right] \mapsto\left[\overline{x_{0}}:-\overline{x_{2}}: \overline{x_{1}}\right]
$$

is orientation preserving, smooth and has exactly one fixed point.

Now let $G$ be a finite cyclic $p$-group. As our theory, we consider $K_{(p)}$, complex $K$-theory localized at $p$.

Lemma 4. Complex K-theory localized at $p$ is $G$ sensitive.

Proof. (cf. [5], p. 33 f.) Let $G=\mathbb{Z} / p^{r}, r \geq 1$. By application of the Gysin sequence, we have an isomorphism

$$
K_{(p)}^{*}(B G) \cong K_{(p)}^{*}[[C]] /\left(e\left(\eta^{p^{r}}\right)\right),
$$

where $C \in K_{(p)}^{2}\left(\mathbb{C} P^{\infty}\right)$ is the Euler class of the universal line bundle on $\mathbb{C} P^{\infty}$ and $e\left(\eta^{p^{r}}\right) \in K_{(p)}^{2}[[C]]$ is the Euler class of the $p^{r}$ th tensor power of this line bundle. One sees that $e\left(\eta^{p^{r}}\right)$ has a factorization containing $C$ and each of the power series

$$
e\left(\eta^{p^{i}}\right) / e\left(\eta^{p^{i-1}}\right), 1 \leq i \leq r .
$$

With help of the formal group law for complex $K$-theory

$$
(x, y) \mapsto x+y+x y,
$$

these power series factors are pairwise coprime, because they all have constant term $p$ and because they are monic polynomials in $C$ of different degrees. In particular, $e\left(\eta^{p^{r}}\right)$ contains a prime factor not contained in any $e\left(\eta^{p^{i}}\right), 0 \leq i<r$. (Here we use 
the fact that $K_{(p)}^{*}[[C]]$ is a unique factorization domain.) The multiplicative set $S$ is generated by the Euler classes

$$
e\left(\eta^{p^{i}}\right), 0 \leq i<r,
$$

hence, by the argument above, $S \cap\left(e\left(\eta^{p^{r}}\right)\right)=\emptyset$. This implies that the localization $S^{-1} K_{(p)}^{*}(B G)$ is indeed different from zero.

By a similar argument, one also sees that $K_{(p)}^{*}$ is not $\mathbb{Z} / n$-sensitive, if $n>1$ is divisible by two different primes $p$ and $q$ : In the localization $S^{-1} K_{(p)}^{*}(\mathbb{Z} / n)$, certain power series in $C$ starting with constant terms $p$ and $q$ are zero and therefore this is also true for some power series starting with 1 . But such a power series is a unit in $K_{(p)}^{*}[[C]]$.

We now additionally assume that $p$ is odd.

Lemma 5. Each oriented (in the usual sense) $G$-manifold $X$ is $\left(K_{(p)}\right)_{G \text {-orientable. }}$

Proof. We prove at first that $(T X)_{G}$ is MSTOP-orientable. Let $(T X)_{G}^{i}$ and $X_{G}^{i}$ be filtrations of $(T X)_{G}$ and $X_{G}$ by finite-dimensional subcomplexes as before. By Kister's theorem [11] we can replace the microbundle $(T X)_{G}^{i}$ over the Euclidean neighbourhood retract $X_{G}^{i}$ by an essentially unique fibre bundle and hence, for each $i$, we get a tautological Thom class

$$
\tau^{i} \in\left[M\left((T X)_{G}^{i}\right), \mathrm{MSTOP}\right]
$$

(which is an MSTOP-cohomology class of the Thom space of $\left.(T X)_{G}^{i}\right)$. The Thom classes constructed in this way are compatible under restriction and so $\left(\tau^{i}\right)$ defines an inverse system, which induces a Thom class for $(T X)_{G}$. Sullivan proved that there is an orientation (cf. [13] or [16])

$$
\operatorname{MSTOP}_{(p)} \rightarrow K_{(p)}
$$

of topological bordism localized at $p$. This completes the proof of Lemma 5 .

Lemmas 4 and 5 together with Theorem 4 complete the proof of Theorem 5 .

With regard to Theorem 3 and the remark following Theorem 1 stated at the beginning of this paper, one might wonder, if under the assumptions of Theorem 3 and in the case that $E$ is $G$-sensitive, one can prove that the induced map

$$
E^{*}\left(N^{G}\right) \rightarrow E^{*}\left(M^{G}\right)
$$

is injective. However, even if we assume that (for compact spaces $X$ ) we have a Künneth formula

$$
S^{-1} E^{*}(B G \times X) \cong E^{*}(X) \otimes_{E^{*}} S^{-1} E^{*}(B G),
$$

such a result is not immediate for general theories $E$, because a noninjective map can very well become an injection after tensoring with some identity map.

\section{ITERATIVE USE OF LOCALIZATION}

Classical Smith theory allows the discussion of Browder's result in the context of Poincaré duality spaces. We use Čech cohomology with $\mathbb{F}_{p}$-coefficients. Let $M$ and $N$ be compact $\mathbb{F}_{p}$-Poincaré duality spaces of the same dimension on which a finite (not necessarily abelian) $p$-group is acting. Furthermore, let $f: M \rightarrow N$ be 
a $G$-equivariant map whose degree (as an element in $\mathbb{F}_{p}$ ) is different from 0 . The ultimate generalization of Browder's theorem would be the conclusion that under these assumptions, the induced map

$$
H^{*}\left(N^{G}\right) \rightarrow H^{*}\left(M^{G}\right)
$$

is injective (which implies that $f^{G}: M^{G} \rightarrow N^{G}$ is surjective, if the space $N$ has good local cohomology properties, e.g. if $N$ is a $\mathbb{F}_{p}$-cohomology manifold). Of course, this is known to be false in general, if we do not assume $G$ to be abelian. On the other hand, in view of Theorem 4, this conclusion is indeed valid (and classical) if $G$ is an elementary abelian $p$-group. But ordinary cohomology (even with integer coefficients) is not $\mathbb{Z} / p^{2}$-sensitive, so we will use induction on the order of $G$ (as Browder does in his proof of Theorem 1) and wish to formulate conditions under which the induced map $H^{*}\left(N^{G}\right) \rightarrow H^{*}\left(M^{G}\right)$ is injective.

The essence of the argument can be seen in the special case $G=\mathbb{Z} / p^{2}$. Let $H<G$ be the subgroup of index $p$ and set $K=G / H \cong \mathbb{Z} / p$. We will use some of the result in [1, Chapter 5.

Considering $f$ as an $H$-equivariant map, one gets an equivariant Gysin homomorphism (which is degree preserving as $m=n$ )

$$
\left(f_{H}\right) !: H_{H}^{*}(M) \rightarrow H_{H}^{*}(N),
$$

where as usual the subscript indicates taking cohomology of the associated Borel constructions. Let $S \subset H^{*}(B H)$ (resp. $T \subset H^{*}(B K)$ ) be the multiplicative subset generated by a nonzero element in $H^{2}(B H)$ (resp. by an nonzero element in $\left.H^{2}(B K)\right)$. We then get a commutative localization diagram

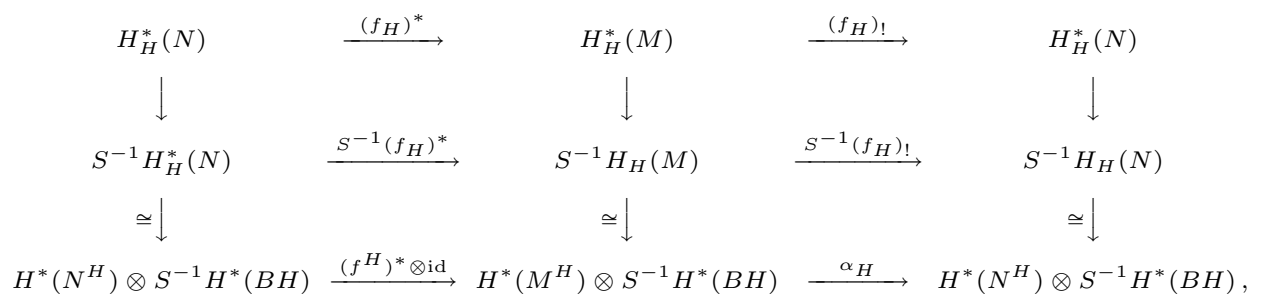

where $\alpha_{H}$ is the composition (mult denotes multiplication from the right)

$$
\text { mult }_{e_{H}^{N}} \circ\left(\left(f^{H}\right) ! \otimes \text { id }\right) \circ \operatorname{mult}_{\left(e_{H}^{M}\right)^{-1}}
$$

using the equivariant Euler classes

$$
e_{H}^{N} \in H^{*}\left(N^{H} \times B H\right), e_{H}^{M} \in H^{*}\left(M^{H} \times B H\right)
$$

of the inclusions $M^{H} \subset M$ and $N^{H} \subset N$ (these Euler classes become invertible after localizing) and the nonequivariant Gysin map for the map $f^{H}$ between $\mathbb{F}_{p^{-}}$ Poincaré duality spaces. Our assumption implies that the first line of this diagram is given by multiplication with a nonzero number in $\mathbb{F}_{p}$ and the same holds for the last line showing that $\left(f^{H}\right)^{*}$ is injective.

For the next step, note that both $N^{H} \times B H$ and $M^{H} \times B H$ carry induced $K$-actions (trivial on the classifying space factors). This suggests taking the corresponding Borel constructions and arguing as before (starting with the last line of the above diagram). In order to make this work, we will assume for the moment 
that the equivariant Euler classes

$$
e_{H}^{M} \in H^{*}\left(M^{H} \times B H\right), e_{H}^{N} \in H^{*}\left(N^{H} \times B H\right)
$$

lie in the image of the maps

$$
\begin{aligned}
H^{*}\left(\left(M^{H}\right)_{K} \times B H\right) & \rightarrow H^{*}\left(M^{H} \times B H\right), \\
H^{*}\left(\left(N^{H}\right)_{K} \times B H\right) & \rightarrow H^{*}\left(N^{H} \times B H\right)
\end{aligned}
$$

induced by restricting to a fibre in the Borel construction. We remark that this lifting assumption is not automatic for the following reason: The $H$-equivariant Euler classes $e_{H}^{M}$ and $e_{H}^{N}$ depend on neighbourhoods of $M^{H}$ in $M$ and $N^{H}$ in $N$. But a priori, an action of the quotient group $K$ can only be defined on $M^{H}$ and $N^{H}$ and not on a neighbourhood of these subsets. However, in some cases this assumption can be verified. We will discuss this point further below.

Let $\tilde{e}_{H}^{N}$ and $\tilde{e}_{H}^{M}$ be preimages $e_{H}^{N}$ and $e_{H}^{M}$ under the maps above. Using a spectral sequence argument, one shows that these classes become again invertible after inverting $S$. We define a map

$$
\alpha_{H}^{\prime}: H^{*}\left(\left(M^{H}\right)_{K} \otimes S^{-1} H^{*}(B H) \rightarrow H^{*}\left(\left(N^{H}\right)_{K}\right) \otimes S^{-1} H^{*}(B H)\right.
$$

as the composition

$$
\operatorname{mult}_{\tilde{e}_{H}^{N}} \circ\left(\left(f^{H}\right) ! \otimes \mathrm{id}\right) \circ \operatorname{mult}_{\left(\tilde{e}_{H}^{M}\right)^{-1}} .
$$

Using the abbreviations $H^{\prime}$ for $S^{-1} H^{*}(B H)$, and $K^{\prime}$ for $T^{-1} H^{*}(B K)$, we then get a commutative diagram

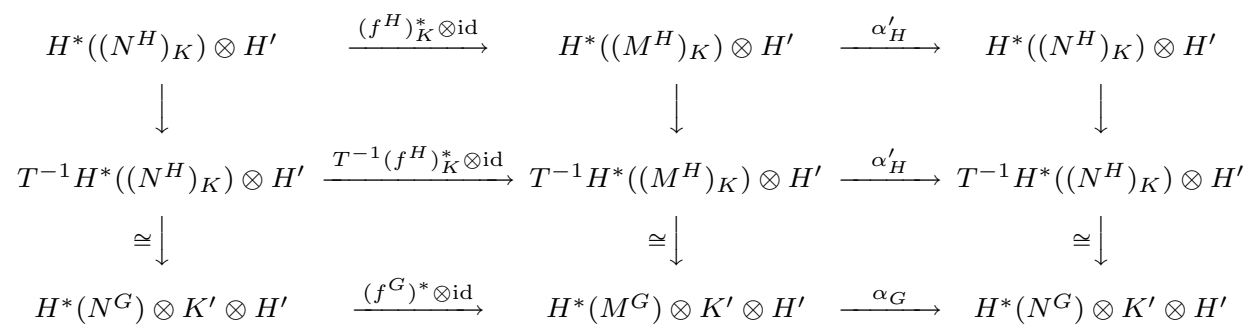

with

$$
\alpha_{G}^{\prime}: \operatorname{mult}_{\tilde{e}_{H}^{N} \cup e_{K}^{N}} \circ\left(\left(f^{H}\right) ! \otimes \mathrm{id}\right) \circ \operatorname{mult}_{\left(\tilde{e}_{H}^{M} \cup e_{K}^{M}\right)^{-1}}
$$

and equivariant Euler classes $e_{K}^{N}, e_{K}^{M}$ of the embeddings of $K$-spaces $N^{G} \subset N^{H}$ and $M^{G} \subset M^{H}$. Using a spectral sequence argument again, the first line of the last diagram is given by multiplication with a nonzero element in $\mathbb{F}_{p}$ and the induced map $H^{*}\left(N^{G}\right) \rightarrow H^{*}\left(M^{G}\right)$ is indeed injective.

Note that the property of $G$ being abelian was not used in the course of this argument. Everything depends on the lifting assumption above which is fairly obvious in the following special case.

Lemma 6. Assume that the induced actions of $K$ on $M^{H}$ and on $N^{H}$ are totally nonhomologous to zero. Then the above lifting assumption for the relevant $H$ equivariant Euler classes is fullfilled (because the relevant maps are surjective in this case).

By imposing strong cohomological retrictions on the actions involved and using the fact that every finite $p$-group is solvable, one can therefore prove the following theorem. 
Theorem 8. Let $M$ and $N$ be compact oriented $\mathbb{F}_{p}$-homology manifolds of the same dimension, let $G$ be a finite p-group and assume the following: The $\mathbb{F}_{p}$-cohomology of $M$ and $N$ is concentrated in even degrees and

$$
p>\max \left\{\operatorname{dim}_{\mathbb{F}_{p}} H^{*}(M), \operatorname{dim}_{\mathbb{F}_{p}} H^{*}(N)\right\} .
$$

Let $f: M \rightarrow N$ be $G$-equivariant. If the degree of $f$ is a nonzero number in $\mathbb{F}_{p}$, then the induced map $M^{G} \rightarrow N^{G}$ is surjective.

Note that by imposing these restrictions, for any normal subgroup $H<G$, the $\mathbb{F}_{p}$-cohomology of $M^{H}$ and $N^{H}$ will be concentrated in even degrees and all the spectral sequences calculating equivariant cohomologies will collapse at the $E_{1}$-level. Now an inductive proof can be carried out as indicated above.

Without these strong additional assumptions, we can prove the lifting assumption for differentiable actions using the following general fact (cf. 3], Proposition $(2.3))$ :

Lemma 7. Let $G$ be a finite abelian p-group and let $H<G$ be a subgroup. Let $E \rightarrow X$ be a real linear $G$-bundle, if $p$ is odd, or a complex linear $G$-bundle, if $p=2$. Each point in $X$ is assumed to have isotropy $H$ and $H$ is assumed to act freely on each fibre. Then the (nonequivariant) Euler class of $E$ lies in the image of

$$
H^{*}(X /(G / H)) \rightarrow H^{*}(X) .
$$

Proof. Note that the claim is obvious if $H$ is a direct factor of $G$, because then the whole bundle carries a free $G / H$-action. In the following, we again concentrate on the special case $G=\mathbb{Z} / p^{2}, H=\mathbb{Z} / p$, the general case being left to the reader. Let $\phi: G \times E \rightarrow E$ be the given $G$-action on $E$. Let $p$ be odd. As an $H$-bundle, $E$ splits as a direct sum

$$
\bigoplus_{j=1}^{\frac{p-1}{2}} E_{j} \otimes V_{j},
$$

where each $E_{j}$ is a real vector bundle and $V_{1}, \ldots, V_{\frac{p-1}{2}}$ are the distinct nontrivial irreducible real $H$-representations. Each $V_{j}$ is a 1-dimensional complex vector space with $H$ acting complex linearly. It follows that the fibrewise $H$-action on $E$ can be propagated to a fibrewise $G$-action $\psi$. The same is true in the case $p=2$ by our additional assumption. In particular, the $H$-action induced by $\psi$ coincides with the former fibrewise $H$-action on $E$.

Now define a new $G$-action on $E$ by

$$
(g, y) \mapsto \psi\left(g^{-1}, \phi(g, y)\right) .
$$

This action and the old $G$-action on $X$ define a new $G$-bundle $E^{\prime} \rightarrow X$. This bundle is nonequivariantly isomorphic to $E \rightarrow X$, but carries an induced free $(G / H)$-action which coincides with the old $(G / H)$-action on the base.

Corollary 9. Let $M$ and $N$ be oriented closed smooth $G$-manifolds, where $G=$ $\mathbb{Z} / p^{2}$. If $p=2$, we assume additionally that the normal bundle of $M^{H}$ in $M$ and of $N^{H}$ in $N$ are complex $G$-bundles. Then the above lifting assumption for the $H$-equivariant Euler classes is fullfilled.

Proof. The fixed point sets $M^{H} \subset M$ and $N^{H} \subset N$ have normal $G$-bundles $\nu^{M} \rightarrow M^{H}$ and $\nu^{N} \rightarrow N^{H}$. The equivariant Euler classes $e_{H}^{M}$ and $e_{H}^{N}$ are nothing 
but the usual Euler classes of the induced vector bundles

$$
\begin{aligned}
\nu^{M} \times_{H} E H & \rightarrow M^{H} \times B H, \\
\nu^{N} \times_{H} E H & \rightarrow M^{H} \times B H .
\end{aligned}
$$

Because $G$ is abelian, these bundles are again $G$-bundles (the $G$-operations are induced by the $G$-operations on $\nu^{M}$ and $\nu^{N}$ ). After taking the cartesian product with the identity $E K \rightarrow E K$, we get induced $G$-bundles

$$
\begin{aligned}
& E K \times\left(\nu^{M} \times_{H} E H\right) \rightarrow E K \times\left(M^{H} \times B H\right), \\
& E K \times\left(\nu^{N} \times_{H} E H\right) \rightarrow E K \times\left(N^{H} \times B H\right),
\end{aligned}
$$

where now $G$ is acting diagonally on the factor $E K$ (using the canonical map $G \rightarrow K$ ) and the other factor. These $G$-vector bundles have the following properties:

- Each point in the base has isotropy $H \subset G$.

- The group $H$ is acting freely on each fibre.

Our claim now follows from Lemma 7

Based on this observation, it is not difficult to set up an inductive proof whose first steps were carried out above, in order to show once again the differentiable case of Browder's theorem.

One could start trying to generalize Lemma 7 in order to prove generalizations of Browder's theorem similar to those discussed in the first part of this paper. However, it seems hard to prove Lemma 7 without the assumption of smoothness of the actions involved.

\section{REFERENCES}

[1] C. Allday, V. Puppe, Cohomological methods in transformation groups, Cambridge Studies in Advanced Mathematics 32 (1993), CUP. MR94g:55009

[2] G.E. Bredon, Fixed point sets of actions on Poincaré duality spaces, Topology 12 (1973), 159-175. MR 48:9708

[3] W. Browder, Pulling back fixed points, Inv. Math. 87 (1987), 331-342. MR88a:57070

[4] T. tom Dieck, Transformation groups, de Gruyter Studies in Mathematics 8 (1987), de Gruyter. MR89c:57048

[5] T. tom Dieck, Kobordismentheorie klassifizierender Räume und Transformationsgruppen, Math. Z. 126 (1972), 31-39. MR 45:7744

[6] E. Dyer, Cohomology theories, Benjamin, New York (1969). MR 42:3780

[7] J. Ewing, R. Stong, Group actions having one fixed point, Math. Z. 191 (1986), 159-164. MR87j:57024

[8] H. Ibisch, Microfibrés normeaux équivariants, C. R. Acad. Sc. Paris Sér A t. 279 (1974), 155-156. MR53:9235

[9] J. Jaworowski, An equivariant extension theorem and $G$-retracts with a finite structure, Manuscripta Math. 35, no. 3, (1981), 323-329. MR83a:57052

[10] K. Kawakubo, Equivariant Riemann-Roch theorems, localization and formal group law, Osaka J. Math. 17 (1980), 531-571. MR81j:57020

[11] J. M. Kister, Microbundles are fibre bundles, Ann. Math. 80 (1964), 190-199. MR31:5216

[12] J. Milnor, Microbundles I, Topology 3, suppl. 1 (1964), 53-81. MR 28:4553b

[13] I. Madsen, R. J. Milgram, The classifying spaces for surgery and cobordism of manifolds, Annals of Mathematics Studies 92 (1979), Princeton University Press. MR81b:57014

[14] S. Mardešić, J. Segal, Shape theory, North Holland (1982). MR84b:55020

[15] G.D. Mostow, Equivariant embeddings in Euclidean space, Ann. of Math. (2) 65 (1957), 432-446. MR 19:291c 
[16] Y. Rudyak, On Thom spectra, orientability and cobordism, Springer Monographs in Mathematics, Springer Verlag (1998). MR99f:55001

[17] R. Switzer, Algebraic topology - homotopy and homology, Grundlehren 212 (1975), Springer Verlag. MR 52:6695

Mathematisches Institut, Universität München, Theresienstr. 39, 80333 München, Germany

E-mail address: Bernhard.Hanke@mathematik.uni-muenchen.de

Fachbereich für Mathematik und Statistik, Universität Konstanz, 78457 Konstanz, GERMANY

E-mail address: Volker.Puppe@uni-konstanz.de 